\title{
Kernos
}

Revue internationale et pluridisciplinaire de religion grecque antique

6 | 1993

Varia

\section{Revue des ouvrages collectifs et mélanges}

\section{(2) OpenEdition}

\section{Journals}

Édition électronique

URL : http://journals.openedition.org/kernos/574

DOI : 10.4000/kernos.574

ISSN : 2034-7871

\section{Éditeur}

Centre international d'étude de la religion grecque antique

Édition imprimée

Date de publication : 1 janvier 1993

Pagination : 398-400

ISSN : 0776-3824

\section{Référence électronique}

"Revue des ouvrages collectifs et mélanges », Kernos [En ligne], 6| 1993, mis en ligne le 07 avril 2011, consulté le 24 septembre 2020. URL : http://journals.openedition.org/kernos/574 ; DOI : https:// doi.org/10.4000/kernos.574 
Fritz GRAF, Heiligtum und Ritual. Das Beispiel der griechisch-römischen Asklepieia , p. 159-199

Madeleine JosT, Sanctuaires ruraux et sanctuaires urbains en Arcadie, p. 205-239

Folkert V AN STRATEN, Votive and Votaries in Greek Sanctuaries, p. 247-284

Roland ÉTIENNE, Autels et sacrifices, p. 291-312

R.A. Tomlinson, Perachora, p. 321-346

La transizione dal Miceneo all'Alto Arcaismo. Dal pallazzo alla città. Atti del Convegno internazionale, Roma, 1419 marzo 1988, Roma, CNR, 1991

Frederick E. BRENK, The Heracles Myth and the Literary Texts Relating to the Myth of Ninurta, p. 507-526, 14 fig.

Giulia PICCAlUGA, Processi di formazione dei miti greci: la fondazione della scrittura, p. 539-548

A. KANTA, Cult, Continuity and the Evidence of Pottery at the Sanctuary of Syme Viannou, Crete, p. 479-506

Walter BURKERT, Typen griechischer Mythen auf dem Hintergrund mykenischer und orientalischer Tradition, p. 527-535

Maria RocCHI, Lineare B e alfabeto nel mito di Palamedes, p. 551-561

\section{Ouvrages collectifs et mélanges}

\section{Christopher A. FARAONE, Dirk OBBINK (éds), Magika Hiera. Ancient} Greek Magic \& Religion, New York, Oxford University Press, 1991, $\mathrm{XIII}+298 \mathrm{p}$.

Christopher A. FARAONE, The Agonistic Context of Early Greek Binding Spells, p. 3-32

J.H.M. STRUBBE, "Cursed be he that moves my bones", p. 33-59

H.S. Versnel, Beyond Cursing: The Appeal to Justice in Judicial Prayers, p. 60-106

Roy KoTANSKY, Incantations and Prayers for Salvation on Inscribed Greek Amulets, p. 107-137

John SCARBoRough, The Pharmacology of Sacred Plants, Herbs, and Roots, p. 138-174

Samson EitRem †, Dreams and Divination in Magical Ritual, p. 175-187

Fritz Graf, Prayer in Magical and Religious Ritual, p. 188-213

John J. W INKLER, The Constraints of Eros, p. 214-243

Hans Dieter BETZ, Magic and Mystery in the Greek Magical Papyri, p. 244259

C.R. PHILliPs III, Nullum Crimen sine Lege: Socioreligious Sanctions on Magic, p. 260-276

Selected Bibliography of Greek Magic and Religion, p. 277-283. 
Marie-Odile GOULET-CAZí, Goulven MADEx, Denis O'BRIEN (éds),

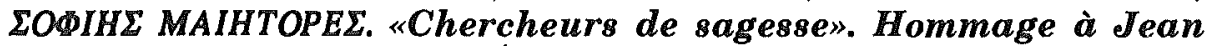
Pépin, Paris, Institut d'Études Augustiniennes-Brepols, 1992, XXXIV+715 p. (Collection d'Études Augustiniennes - Série Antiquité, 131).

Marie-Odile GoulET-CAZÉ, L'Ajax et l'Ulysse d'Antisthène, p. 5-36

Jean-Marie Flamand, Deus otiosus. Recherches lexicales pour servir $\grave{a}$ l'histoire de la critique religieuse d'Epicure, p. 147-166

Lambros Couloubaritsis, Le logos hénologique chez Plotin, p. 231-243

Pier Franco Beatrice, Towards a new edition of Porphyry's fragments against the Christians, p. 347-355

Goulven MADEC, Augustin et Prophyre. Ebauche d'un bilan des recherches et des conjectures, p. 367-382

Coloman ViolA, “... hoc est enim Deo esse, quod est magnum esse". Approche augustinienne de la grandeur divine, p. 403-420

Henri Dominique SAFFREY, Le thème du malheur des temps chez les derniers philosophes néoplatoniciens, p. 421-431

Luc BRISSON, Le corps "dionysiaque». L'anthropogonie décrite dans le Commentaire sur le Phédon de Platon (1, par. 3-6) attribué à Olympiodore est-elle orphique?, p. 481-499

Ysabel DE ANDIA, Philosophie et union mystique chez le Pseudo-Denys l'Aréopagite, p. 511-531

Sylvain MATTON, L'intégration en Christ des dieux antiques selon Georges de Venise. Théologie cosmique et théologie chrétienne à la Renaissance, p. 651-676

Serta Leodiensia Secunda. Mélanges publiés par les Classiques de Liège à l'occasion du 175 e anniversaire de l'Université, Université de Liège, Département des Sciences de l'Antiquité, C.I.P.L., 1992, XVIII+468 p.

Léon LACROIX, Thésée et Aithra sur la coupe de Macron, p. 247-256

André MOTTE, Le premier pèlerinage delphique, p. 367-376

\section{Contributions particulières}

P. ANGELINI BerNARDINI, Mythe et agon : Héraclès fonateur des jeux olympiques, in R. Renson, M. LÄMmer, J. RIORDAN, D. Chassiotis (éds), The Olympic Games through the Ages: Greek Antiquity and its Impact on Modern Sport. Proceedings of the 13th international HISPA Congress Olympia/Greece, May 22-28, 1989, Athens, 1991, p. 13-22.

Giovanni Casadio, The Manichaean Metempsychosis: Typology and Historical Roots, in G. WIEßNER, H.J. KLIM KEIT (éds), Studia Manichaica. II. Internationaler Kongreß zum Manichäismus, 6.-10. August 1989 St. Augustin/ Bonn, Wiesbaden, Harrassowitz, 1992, p. 105-130. 
-, Donna e simboli femminili nella gnosi del II secolo, in La donna nel pensiero antico, Genova, Marietti, 1992, p. 305-329.

Florence DoYEN, R. PREYs, La présence grecque en Égypte ptolémalque, les traces d'une rencontre, in M. BROzE, Ph. TALON (éds), L'atelier de l'orfêvre. Mélanges offerts à Ph. Derchain, Leuven, Peeters, 1992 (Coll. de l'IPHO «Lettres orientales», 1), p. 63-85.

René LeBrun, De quelques cultes lyciens et pamphyliens, in Hittite and other Anatolian and Near Eastern Studies in Honor of Sedat Alp, Ankara, 1992, p. 357-363.

J. Redfield, From Sex to Politics: The Rites of Artemis Triklaria and Dionysos Aisymnetes at Patras, in D.M. HALPERIN, J.J. WinkLER, F.I. ZEITLIN (éds), Before Sexuality: The Construction of Erotic Experience in the Ancient Greek World, Princeton Univ. Press, 1990, p. 115-134.

Maria RocCHI, Les oracles des Pisistratides dans le temple d'Athéna, in C. BAURAIN, C. Bonnet, V. KRINGS (éds), Phoinikeia grammata. Lire et écrire en Méditerranée. Actes du Colloque de Liège, 15-18 novembre 1989, Namur, Société des études classiques, 1991 (Studia Phoenicia - Coll. d'études classiques, 6), p. 577-589.

P. Roesch, Les cultes égyptiens en Béotie, in L. Criscuolo, G. GeraCi, Egitto e Storia dall'Ellenismo all'Età Araba. Bilancio di un confronto, Bologna, 1989, p. 621-629.

Suzanne SAÏD, Le mythe de l'Aréopage avant la Constitution d'Athènes, in Aristote et Athènes. Fribourg (Suisse), 23-25 mai 1991, études rassemblées par M. PiÉrart, Paris, de Boccard, 1993, p. 155-184

K. Tzavélla-Evjen, Heroes who do not "Rest in Peace": Legends and the Archaeological Record, in J.M. FossEY, J. MoRIN (éds), Boeotia Antiqua II. Papers on recent work in Boiotian Archaeology and Epigraphy, Amsterdam, Gieben, 1992 (McGill Univ. Monographs in Classical Archaeology and History, 11)

A. VENERI, Posidone e l'Elicone: alcune osservazioni sull'antichità e la continuità di una tradizione mitica beotica, in A. SCHACHTER (éd.), Essays in the topography, history and culture of Boiotia, Montréal, 1990 (Teiresias Supplement, 3), p. 129134.

K.-W. WELWEI, Heroenkult und Gefallenenehrung im antiken Griechenland, in G. BINDER, B. EFFE (éds), Tod und Jenseits im Altertum, Trier, Wissenschaftlicher Verlag Trier, 1991, p. 50-70.

\section{Rééditions, réimpressions et traductions}

Bruit Z aidman Louise, Schmitt Pantel Pauline, Religion in the Ancient Greek City, transl. by Paul Cartledge, Cambridge Univ. Press, $1992, \mathrm{xX}+278$ p. ISBN : 0-521-42357-0.

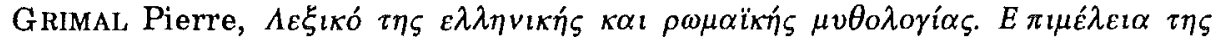

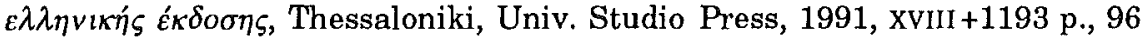
fig. [éd. or. 1976, P.U.F.]. 